\title{
Indoor path visualization method based on the spatial characteristics of indoor environment
}

\author{
Jiafeng Shi ${ }^{\mathrm{a}, \mathrm{b}, \mathrm{c}}$, Jie Shen ${ }^{\mathrm{a}, \mathrm{b}, \mathrm{c}, *}$, Zdeněk Stachoň ${ }^{\mathrm{d}}$, Yawei Chen ${ }^{\mathrm{a}, \mathrm{b}, \mathrm{c}}$ \\ ${ }^{a}$ Key Laboratory of Virtual Geographic Environment (Nanjing Normal University), Ministry of Education, Nanjing 210023, China; \\ jiafeng.shi@outlook.com(J.S.), shenjie@njnu.edu.cn(J.S),chen1546362828@gmail.com(Y.C) \\ ${ }^{b}$ Jiangsu Center for Collaborative Innovation in Geographical Information Resource Development and Application, Nanjing \\ 210023, China; \\ ${ }^{c}$ School of Geography Science, Nanjing Normal University, Nanjing 210023, China \\ ${ }^{d}$ Department of Geography, Faculty of Science, Masaryk University, Brno, Czech Republic; zstachon@geogr.muni.cz (Z.S.) \\ * Corresponding author
}

Keywords: Indoor map, Indoor spatial characteristics, Path visualization, Map design

\begin{abstract}
:
With the increasing number of large buildings and more frequent indoor activities, indoor location-based service has expanded. Due to the complicated internal passages of large public buildings and the three-dimensional interlacing, it is difficult for users to quickly reach the destination, the demand of indoor paths visualization increases. Isikdag (2013), Zhang Shaoping (2017), Huang Kejia (2018) provided navigation services for users based on path planning algorithm. In terms of indoor path visualization, Nossum (2011) proposed a "Tubes" map design method, which superimposed the channel information of different floors on the same plane by simplifying the indoor corridor and the room. Lorenz et al (2013) focused on map perspective (2D/3D) and landmarks, developed and investigated cartographic methods for effective route guidance in indoor environments. Holscher et al (2007) emphasized using the landmark objects at the important decision points of the route in indoor map design. The existing studies mainly focused on two-dimensional plane to visualize the indoor path, lacking the analysis of three-dimensional connectivity in indoor space, which makes the intuitiveness and interactivity of path visualization greatly compromised. Therefore, it is difficult to satisfy the wayfinding requirements of the indoor multi-layer continuous space. In order to solve this problem, this paper aims to study the characteristics of the indoor environment and propose a path visualization method. The following questions are addressed in this study: 1) What are the key characteristics of the indoor environment compared to the outdoor space? 2) How to visualize the indoor paths to satisfy the users' wayfinding needs?
\end{abstract}

Compared to the outdoor environment, the indoor environment has specific spatial characteristics:

1) For the space division boundary, the outdoor paths have clear boundary contrasting with other elements, users can usually use only the planned network. But in the individual-oriented indoor space, users can move freely in the passable area.

2) The indoor space has not only a horizontal dimension but also a vertical and oblique dimension with possibilities of movements in different directions. That is increases the difficulty for users' wayfinding in the indoor multi-layer continuous space.

3) For the functional attribute of indoor path, it is different from outdoor road such as single-line, two-way traffic lines, etc. The functional attribute of indoor path can be divided into staff channel, VIP channel, disabled channel, and an open channel for the public.

4) For the semantic structure, the indoor path has usually no name or direction, and it is more difficult for users to describe the indoor path.

According to the indoor path spatial characteristics, and combined with the map design theory, this study proposes a path visualization method for indoor map design: 1) Divided indoor paths boundary into two types: passable and impassable. Using solid line to indicate the impassable boundary, and dotted line indicate the passable boundary. 2) Fully consider the physical connect position and direction of the straight ladder and escalator in the floor conversion. Using 2D and 2.5D method to visually display each floor information and path conversion position. 3) Each function indoor path is visualized by using different colour polygon, and design symbols to emphasis the special paths (such as VIP, disable channel, etc.). 4) Combine the important entities around the path and add the symbols on it, to help the user describe the location of the path. In summary, we select a large shopping mall as an example, according to the comparative analysis of user experiments, the feasibility and suitability of the indoor path expression method in this study were verified, which provide a reference for indoor map design. 
This study is still limited to static indoor maps, we hope to further explore the indoor path expression method based on multi-device display (such as touch screens, interactive, web applications, etc.), and how to express paths for indoor navigation system.

\section{Reference}

Holscher C, Buchner S J, Brosamle M, et al. Signs and maps-Cognitive economy in the use of external aids for indoor navigation[C]//Proceedings of the Annual Meeting of the Cognitive Science Society. 2007, 29(29).

Huang Kejia, Li Shaojie, Zuo Yao, et al. Design and Implementation of Cross-floor Route Planning Technology Based on Indoor Road Network[J]. GEOMATICS WORLD, 2018,25(03):91-95.

Isikdag U, Zlatanova S, Underwood J. A BIM-Oriented Model for supporting indoor navigation requirements[J]. Computers, Environment and Urban Systems, 2013, 41: 112-123.

Lorenz A, Thierbach C, Baur N, et al. Map design aspects, route complexity, or social background? Factors influencing user satisfaction with indoor navigation maps[J]. Cartography and Geographic Information Science, 2013, 40(3): 201-209.

Nossum A S. IndoorTubes a novel design for indoor maps[J]. Cartography and Geographic Information Science, 2011, 38(2): 192-200.

Ohm C, Müller M, Ludwig B. Displaying landmarks and the user's surroundings in indoor pedestrian navigation systems[J]. Journal of Ambient Intelligence and Smart Environments, 2015, 7(5): 635-657.

Zhang Shaopin. Dynamic path planning and design of building emergency evacuation for mobile terminal[D] Fujian Normal University,2017. 